\title{
How to overcome challenges and find strength from within: Resilience and Gerontology
}

Resilience, as a psychological concept, has gained an importance over the past few years that goes far beyond the academic sphere. Notably, the concept has been employed in international debates in the context of urbanization, environmental disasters and other crises, and increasingly refers not only to individuals, but also to communities and environments. It is applicable to the many contexts in which individuals, communities or systems need to adapt to new conditions without losing their functionality.

The concept of resilience is therefore closely linked to geriatrics and gerontology. Over the life course, and especially in old age, people need to compensate for losses, overcome challenges, and adapt to physical, mental, environmental and social changes. The same applies to communities, which, for example, as a result of population ageing, face new challenges. One example would be the need to adapt public spaces to a society with more older people and fewer children. It therefore seems almost impossible to study geriatrics and gerontology without understanding the concept of resilience.

There are also close ties between resilience and gerontological concepts such as the idea of active ageing and its fundamental capitals (vital capital, social capital, finance capital and knowledge capital). It is these reserves that are necessary to adapt and learn from the challenges one encounters throughout life, and which make a person or a community more resilient. Thus, the concept of resilience is indispensable to understanding active ageing as the process of optimizing opportunities for health, participation, lifelong learning and security to improve, or maintain, quality of life as people age (ILC-Brazil 2015).

With increasing longevity in contexts of increasingly rapid and abrupt change, mainly related to digitalization, building resilience during the life course is essential to ensure well-being and quality of life in old age. The mantra of "the sooner the better, it is never too late" also applies to the building of resilience. We must invest in creating resilience at all ages and levels.

The 4th Industrial Revolution, which is characterized by a disconcerting new fusion of technologies in the digital, physical and biological domains, causes disruptive changes. Those who lack resources, including the intellectual capital and, for example, the mental stability to cope with and adapt to these changes will become excluded. In contrast, someone who is used to dealing with technological changes and who has accumulated financial, social and intellectual resources along the life course will overcome the challenges more easily. 
The discussion of the issue of resilience is also necessary today because of the many crises that the world, and in particular Brazil, are experiencing. A glance at the newspaper reveals numerous situations requiring resilience: violence in Brazil's urban centers occurring alongside terrorism in Europe, wars and humanitarian crises in the Middle East and Africa, and growing populism and xenophobia in the USA and Europe.

In short, the challenges we encounter in life are constant and cover all areas of life, from health and security to social participation and lifelong learning. Some will use barriers, whether big or small, as a spring board, while others will need more support to overcome these challenges and to be able to see them as challenges and not as limits.

It is in this context that the Fifth International Longevity Forum will address the theme of building resilience across the life course. To overcome the challenges created by the complex relationship between population ageing, digitalization and the risks set out above, individual resilience must be created throughout life, as well as at the community and society level. The Fifth Forum, which takes place on October 19 and 20 in Rio de Janeiro, brings together speakers from Brazil and other countries from various fields, including psychology, sociology, demography, gerontology, geriatrics, nursing, architecture and design. As in other years, the content from the Forum will be summarized in a succinct and useful document to promote core ideas about resilience in an increasingly long-lived world.

Ina Voelcker, Technical Director, International Longevity Centre Brazil (ILC-Brazil) 